\title{
APPLYING THE WAVELET TRANSFORM TO STUDY THE FEATURES OF FREAK WAVES
}

\author{
Li-Chung $\mathrm{Wu}^{1}$, Beng-Chun $\mathrm{Lee}^{2}$, Chia Chuen Kao ${ }^{1}$, Dong-Jiing Doong ${ }^{3}$, Chih-Chiang Chang ${ }^{1}$ \\ The issues of freak waves are more and more popular since the late 1980s. This study tries to use the wavelet \\ scalogram of freak wave records to investigate the energy characteristics during the occurrence of the freak waves. \\ Through the analysis of the wave energy and phase, it is found that as freak waves occur, the component waves will \\ lead to constructive superposition due to similar phases. The wavelet scalogram provides the other idea to explain the \\ features of freak waves.
}

Keywords: freak waves; wavelet transform; wavelet scalogram

\section{INTRODUCTION}

Freak waves are defined as wave whose heights are more than twice the significant wave height (Chalikov 2009). At present, the phenomenon of freak wave is still a matter of active research. Due to their danger to various ocean actives, many studies tried to find out mechanisms of freak waves by different methods, so as to decrease the damage from freak wave events. For example, Onorato et al. (2001) studied freak waves generation in a random sea state characterized by the Joint North Sea Wave Project (JONSWAP) spectrum. They revealed how freak waves in a random sea state are more likely to occur for large values of the Phillips parameter and the enhancement coefficient showed from extensive numerical simulations of the nonlinear Schrödinger equation. Gramstad and Trulsen (2010) applied numerical Monte Carlo simulations to discuss the features of freak waves. They found evidence that a realistic short-crested wind sea can on average experience a small increase in freak wave probability.

In addition to numerical simulation, the in-situ measurement of ocean waves can help study the features of freak waves too. Field measurements must be performed to increase practical knowledge of waves. However, most observation sensors are suitable for application nearshore or in shallow water areas. Apart from remote sensing devices, buoys and vessels are the only platforms to satisfy wave measurement in deep water areas (Tucker and Pitt, 2001). In-situ wave acceleration records measured from data buoy can be transformed into the acceleration spectrum by the suitable spectrum transformation method. Wave spectrum, which can be obtained from the acceleration spectrum by the transfer function, should be an ideal tool to discuss the features of freak waves in frequency domain. The Fourier transform was often used in the past to calculate the spectral information from the acceleration records. For the algorithm of the Fourier transform, stationarity within the observed period was assumed. However, it is a fact that most real signals in nature should be non-stationary; as are natural wave signals. If the algorithm of Fourier transform is used in the wave analysis, what can be perceived is just the average energy of this section on the frequency spectrum, and the instantaneous energy structure, in fact, can not be viewed from the Fourier spectrum.

The wavelet transform method has increased its applications in recent years since its inception in the early 1980s. The wavelet transform is now recognized as a useful, flexible, and efficient technique to analyze non-stationary signals as well as wave records which are obtained from experimental or insitu measurements. The wavelet scalogram, obtained from the wavelet transform of wave records, presents wave features in time and also frequency domain. It should be a proper tool to observe instantaneous energy structure from the freak wave events. Thus, this research tries to discuss the features of the freak wave events through the wavelet scalogram and the instantaneous energy structures for freak waves to occur.

\section{THEORETICAL PRELIMINARIES}

Based on the theory of one dimensional continuous wavelet transform, the acceleration signal can be broken into various wavelets which are scaled and shifted versions of a pre-chosen mother wavelet function. The acceleration signal $A_{c}(t)$ corresponds to the acceleration value of each time point $t$. The

\footnotetext{
${ }^{1}$ National Cheng Kung University, Tainan, Taiwan, 70101, ROC

${ }^{2}$ Huafan University, Taipei County, Taiwan, 22301, ROC

${ }^{3}$ National Taiwan Ocean University, Keelung ,Taiwan, 20224, ROC
} 
continuous wavelet transform $W_{A c}(b, a)$ of acceleration signal $A_{c}(t)$ for a transformed mother wavelet $\psi_{b, a}$ is:

$$
W_{A c}(b, a)=\frac{1}{\sqrt{C_{\psi}}}\left\langle\psi_{b, a} \mid A_{c}(t)\right\rangle
$$

in which the scaling parameter $a$ is related to the dilated frequency in the time domain. The nondimensional scale factor $a$ gives all dilated versions of the mother wavelet the same energy, that is, it is the ratio of the size of the dilated wavelet to the size of the mother wavelet. The translation factor $b$ corresponds to the position of the wavelet function as it shifts through the whole time domain. The mother wavelet function $\psi_{b, a}$ must satisfy two mathematical properties to be clasfied as 'wavelets'. First, they must have must have finite energy:

$$
\left\|A_{c}\right\|^{2}=\int_{R^{2}}\left|A_{c}(t)\right|^{2} d t<\infty
$$

Second, they must have an "admissibility condition”, which can be expressed by:

$$
C_{\psi}=(2 \pi) \int_{-\infty}^{\infty} \frac{|\hat{\psi}(\omega)|^{2}}{|\omega|} d \omega<\infty
$$

in which the function $\hat{\psi}$ is the Fourier space of function $\psi$; it means the function in frequency domain. $\omega$ is the frequency. Eq. (1) can also be expressed as:

$$
\begin{gathered}
W_{A c}(b, a)=C_{\psi}^{-1 / 2} a^{-1 / 2} \int \psi_{b, a}^{*}(t) \cdot A_{c}(t) d t \\
\psi_{b, a}(t)=\frac{1}{\sqrt{a}} \psi\left(\frac{t-b}{a}\right)
\end{gathered}
$$

where $\psi^{*}$ is the complex conjugate of the wavelet function $\psi . W_{A c}(b, a)$ conserves the norm of the signal, thus its total energy (Buessow 2007):

$$
E_{n}=\int\left|A_{c}(t)\right|^{2} d t=\iint\left|W_{A c}(b, a)\right|^{2} \frac{d a d b}{a^{2}}
$$

To implement the Equations above, it is necessary to choose a mother wavelet function $\psi$ first. The Morlet wavelet function, which is a popular wavelet function used in many applications, is chosen here for detecting the instantaneous wave information from the acceleration signal. The Morlet mother wavelet function and its function in the Fourier (spectral) space, as defined in Eqs. (7) and (8), were used throughout the implementation procedures in this study.

$$
\begin{gathered}
\psi(t)=e^{\left(i \omega_{0} t\right)} \cdot e^{\left(-0.5 t^{2}\right)} \\
\hat{\psi}(\omega)=(2 \pi)^{-0.5} \cdot e^{-0.5\left(\omega-\omega_{0}\right)^{2}}
\end{gathered}
$$

in which $\omega_{0}$ is a constant that forces the admissibility condition , as shown in Eq. (3), to be satisfied. It was suggested to set up as 5.5 following the previous study (Jordan et al. 1997). For calculating the sea surface elevation time series $\eta(t)$ from the energy, the inverse wavelet transform is applied here:

$$
\eta(t)=\frac{1}{C_{\psi}} \int_{0}^{\infty} \int_{-\infty}^{\infty} W_{\eta}(b, a) \cdot \psi_{b, a}(t) d b \frac{d a}{a^{2}}
$$


where $W_{\eta}(b, a)$ is the continuous wavelet transform of the sea surface elevation time series $\eta(t)$, it is related to $W_{A c}(b, a) . W_{A c}(b, a)$ presents the energy features in the domain of parameters $a$ and $b$, it is related to the function of sea elevation. A regular wave in the time domain, the water level could be given by:

$$
\eta(t)=P \cdot \cos (2 \pi f t+\varepsilon)
$$

in which $P$ is the wave amplitude, $f$ is the frequency, and $\varepsilon$ is the phase of the wave component. The sea surface elevation is the double integral of the wave acceleration signal $A_{c}(t)$. In other words, $A_{c}(t)$ is the double differential of $\eta(t)$ :

$$
A_{c}(t)=\left(\eta(t) \frac{1}{d t}\right) \frac{1}{d t}=-(2 \pi f)^{2} P \cdot \cos (2 \pi f t+\varepsilon)=-(2 \pi f)^{2} \eta(t)
$$

As shown in Eq. (11), the transfer function between the wave acceleration and water level function should be $-(2 \pi f)^{2}$ :

$$
\eta(t)=-(2 \pi f)^{-2} A_{c}(t)
$$

As shown in Eq. (4), the relationship between $W_{A c}(b, a)$ and $A_{c}(t)$ has been revealed. Similar to Eq. (4), the relationship between $W_{\eta}(b, a)$ and $\eta(t)$ was shown here:

$$
W_{\eta}(b, a)=C_{\psi}^{-1 / 2} a^{-1 / 2} \int \psi_{b, a}^{*}(t) \cdot \eta(t) d t
$$

Combining Eqs. (4), (12) and (13), we can calculate the wavelet coefficient of the sea surface elevation from the wavelet coefficient of the wave acceleration signals:

$$
W_{\eta}(b, a)=-(2 \pi f)^{-2} \cdot W_{A c}(b, a)
$$

Therefore, the sea surface elevation can be obtained from the wave acceleration records by Eqs. (9) and (14). The wavelet scalogram is called $\left|W_{\eta}(b, a)\right|^{2}$ (Yeh and Liu 2008), the scalogram is a measure of the energy distribution over time shift factor $b$ and scaling factor $a$ of the signal. According to the law of the conservation of energy, the relationship between the water level and wavelet scalogram is shown here:

$$
E=\int_{-\infty}^{\infty}|\eta(t)|^{2} d t=\int_{-\infty}^{\infty} \int_{-\infty}^{\infty}\left|W_{\eta}(b, a)\right|^{2} \frac{d a d b}{a^{2}}
$$

The transfer function between the wavelet scalogram $\left|W_{n}(b, a)\right|^{2}$ and $\left|W_{A c}(b, a)\right|^{2}$ is shown here:

$$
\left|W_{\eta}(b, a)\right|^{2}=(2 \pi f)^{-4} \cdot\left|W_{A c}(b, a)\right|^{2}
$$

To apply Eqs. (9), (14) and (16), we can obtain sea surface elevation from the acceleration signal $A_{c}(t)$. The flow chart of the whole algorithm described above is shown in Fig. 1. 


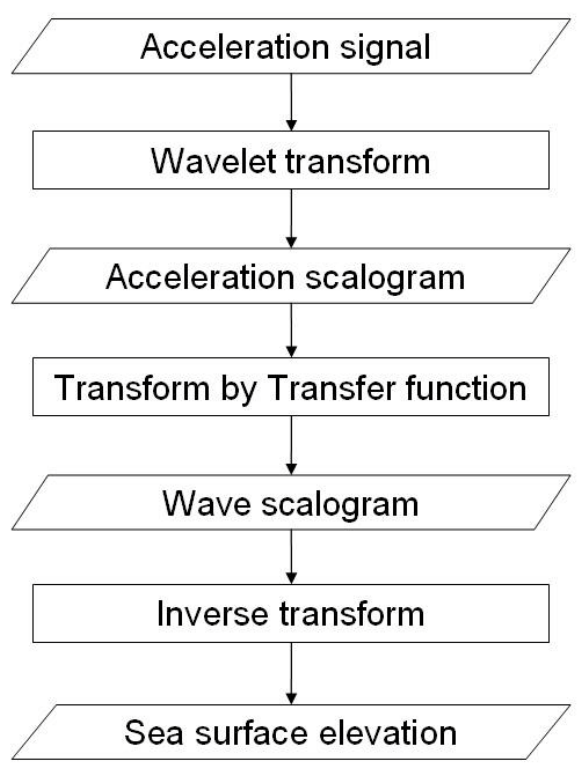

Figure 1. The flow chart to derive wave scalogram and sea surface elevation.

\section{VERIFICATION OF THE WAVELET ALGORITHM}

To understand the feasibility of wavelet scalogram and sea surface elevation from the nonstationary acceleration signal, here we simulated the wave signals in the time domain:

$$
\eta(t)= \begin{cases}P_{1} \cdot \cos \left(2 \pi f_{1} t\right) & t \leq T / 2 \\ P_{2} \cdot \cos \left(2 \pi f_{2} t\right) & t>T / 2\end{cases}
$$

in which $T$ is the total time duration of the sea surface elevation, the definitions of other parameters are the same as those in Eq. (10). In Eq. (17), $P_{1}=1 \mathrm{~m}, f_{1}=0.1 \mathrm{~Hz}, P_{2}=0.5 \mathrm{~m}$ and $f_{2}=0.2 \mathrm{~Hz}$ are used to simulate two different kinds of wave system. To analyze discretely sampled data, such as the wave records from the wave sensor, it is necessary to discretize the sea surface elevation function. A simplified example as shown in Eq. (18) could be introduced to explain the idea:

$$
T=N_{t} \Delta t
$$

The discrete velocity and acceleration of the sea surface elevation can be obtained by:

$$
\begin{gathered}
V_{e}\left(m_{t}\right)=\left[\eta\left(m_{t}\right)-\eta\left(m_{t}-1\right)\right] / \Delta t \quad m_{t}=2, \ldots ., N_{t} \\
A_{c}\left(n_{t}\right)=\left[V_{e}\left(n_{t}\right)-V_{e}\left(n_{t}-1\right)\right] / \Delta t \quad n_{t}=2, \ldots ., N_{t}-1
\end{gathered}
$$

For this study, the sampling rate of wave data, both from simulation and in-situ observed data, are $2 \mathrm{~Hz}$. This means $\Delta t=0.5 \mathrm{sec}$ in the cases of study. The data samples are 1024 points for every case of the study. In other words, the duration of wave data records would be $512 \mathrm{sec}$. Fig. 2(a) shows the wavelet scalogram of the wave signals calculated by the theories of wavelet transform and Eq. (14) from the simulated water level signal. Because of the capability for time-frequency analysis (Lee et al. 2007), the scalogram presented the energy of the signals distributed over the time-frequency domain. The spectral characteristics in different time points from the whole time domain are presented by the wavelet scalogram. As shown in Fig. 2(a), obvious energy is concentrated in the very low frequency band. This is influenced by the transfer function $-(2 \pi f)^{-2}$ which was described in Eq. (14). This energy on the very low frequency band is seen as noise; is necessary to remove the energy from the very low frequency band from the wavelet scalogram. A filter with a cut off frequency of $0.03 \mathrm{~Hz}$ was used to eliminate the low frequency noise (Wang et al. 1993). Fig. 2(b) shows the wavelet scalogram result after removing the noise from the low frequency band. The energy distribution from Fig. 2(b) is 
concentrated on the frequency bands of $0.1 \mathrm{~Hz}$ during the first half of the scalogram in the time domain, and is concentrated on the frequency bands of $0.2 \mathrm{~Hz}$ during the latter half of the scalogram. This agrees with the input conditions of the simulated wave signals.
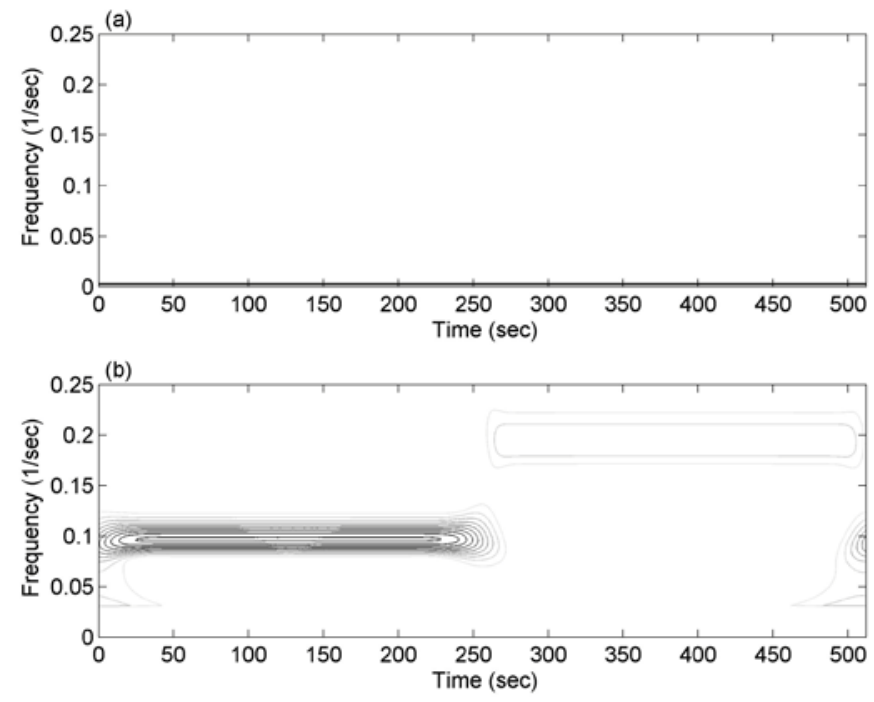

Figure 2. (a). The wavelet scalogram of wave signals which are calculated by the wavelet transform; (b). The wavelet scalogram result after eliminating the noise from low frequency band.

\section{WAVELET SCALOGRAM OF THE FREAK WAVES}

Here we calculate the wavelet scalogram from the natural ocean wave signals. The acceleration signals are collected from the Hualien sea area in the eastern part of Taiwan (Fig. 3). This sea area lies on the border between the largest land mass and the largest ocean in the world. As a result, the marine environments here are complex and sensitive.

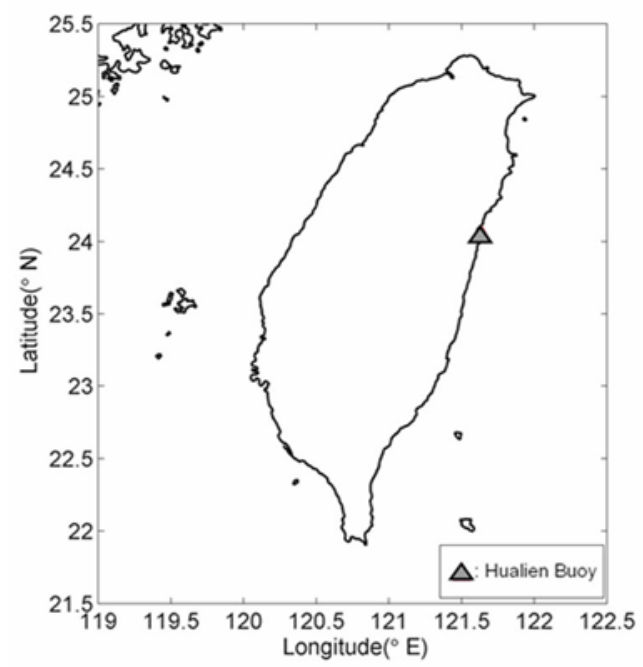

Figure 3. Location of in-situ wave stations in Taiwan.

Figure 4 shows an example of wavelet scalogram and its sea surface elevation. It is revealed that the positions where the maximum wave energy from the wavelet scalogram are often positions of freak wave events. In addition to observe the features of energy, the wavelet scalogram also provides the information of wave phase. Based on the mathematical characteristics of Morlet Wavelet function, the function $W_{\eta}(b, a)$ should be a complex number. It is possible to obtain the phase information by: 


$$
\varepsilon=\tan ^{-1}\left(\operatorname{Im}\left(W_{\eta}(b, a)\right) / \operatorname{Re}\left(W_{\eta}(b, a)\right)\right)
$$

where $\varepsilon$ is the phase angle of wave components. Hence, we can transform the scalogram into the phase spectra. As shown in Fig. 5, the phase angles from the phase spectra are quite similar around the frequency bins of peak frequency from most of the freak wave cases. Due to the similar phase angles from different frequency bins, the sea surface elevation may form an extreme wave. This should be one of the factors to result in freak waves. We also discuss the instantaneous phase spectra before and after before the occurrence of freak wave. The results show that the phase distributions in Fig.6 and Fig.7 are more dispersive than that in Fig.5. This result explains why the freak events are often instantaneous. The results of wavelet scalogram and phase spectra present the difference of phase distributions between freak wave and non-freak wave events. Our results provide the other idea to understand the feature of freak waves.
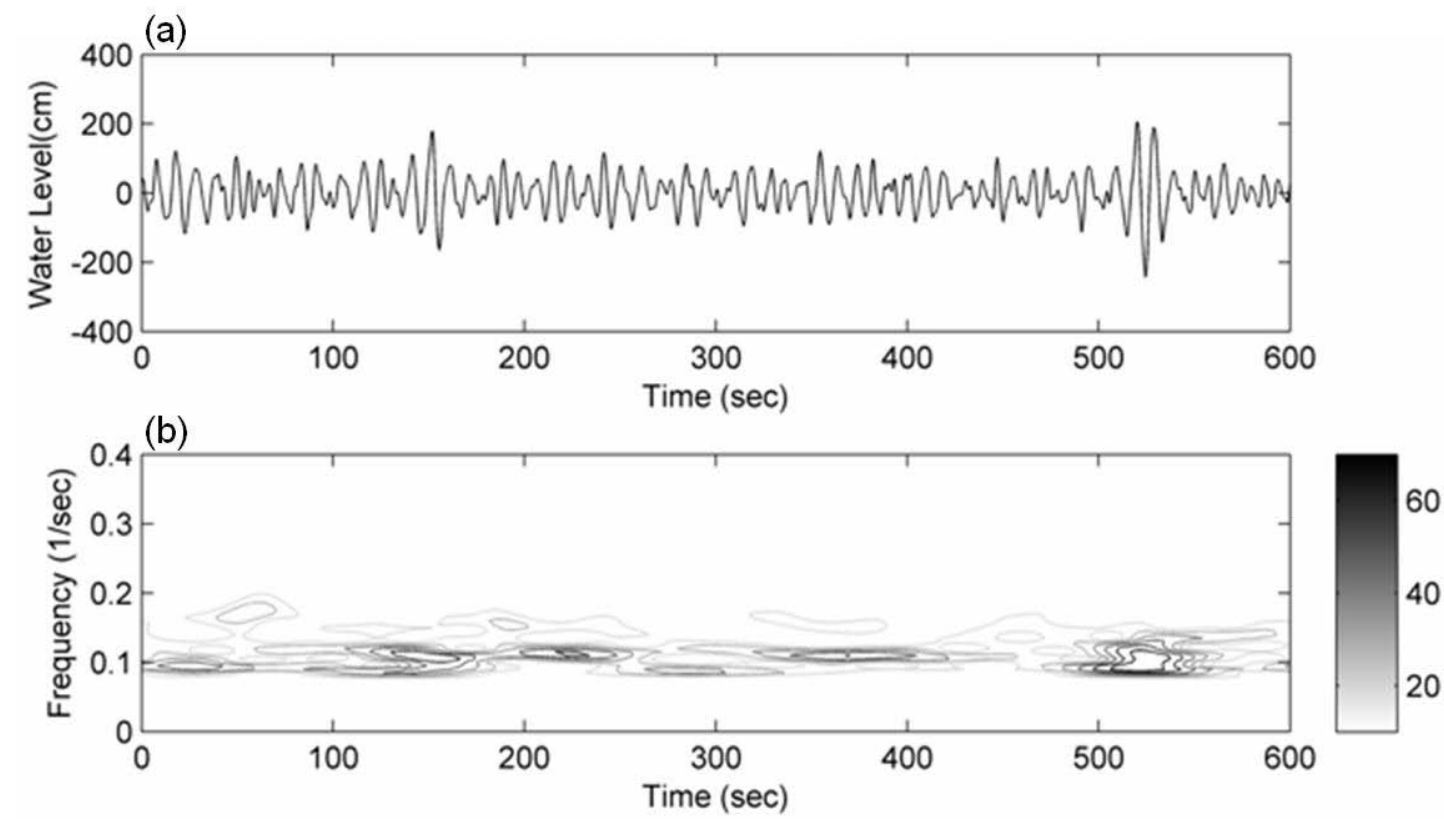

Figure 4. (a).Water level time series; (b). Wavelet scalogram.

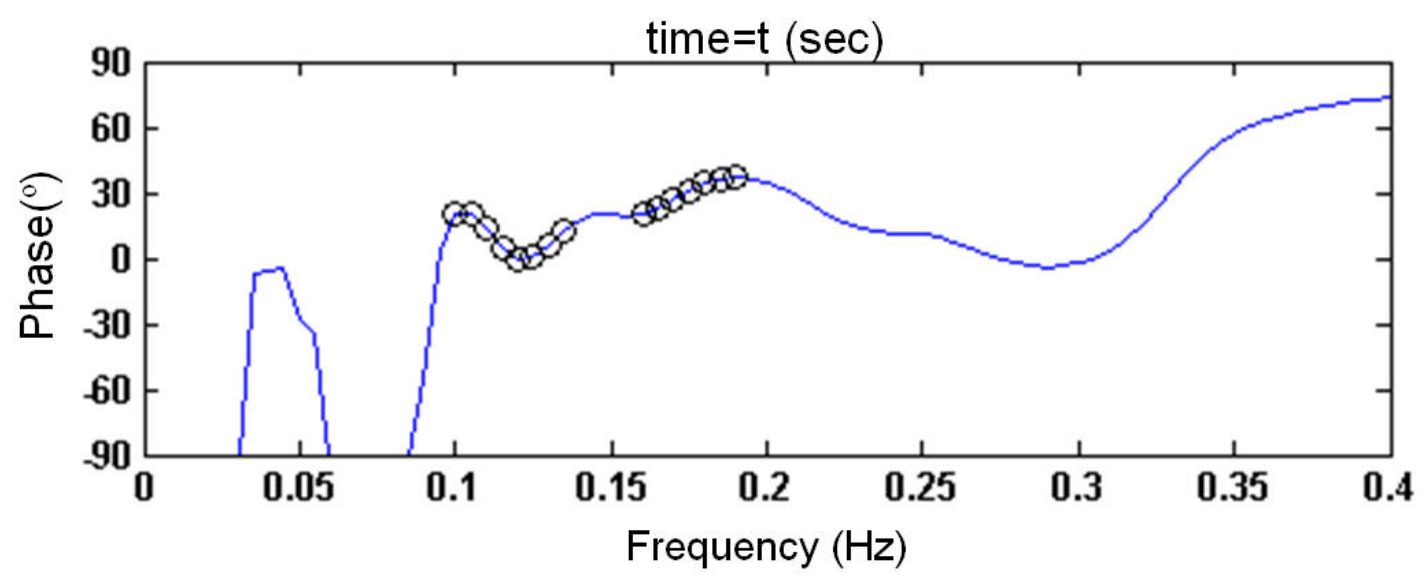

Figure 5. The phase spectrum analyzed by the wavelet algorithm: The instantaneous phase spectrum of freak wave. 

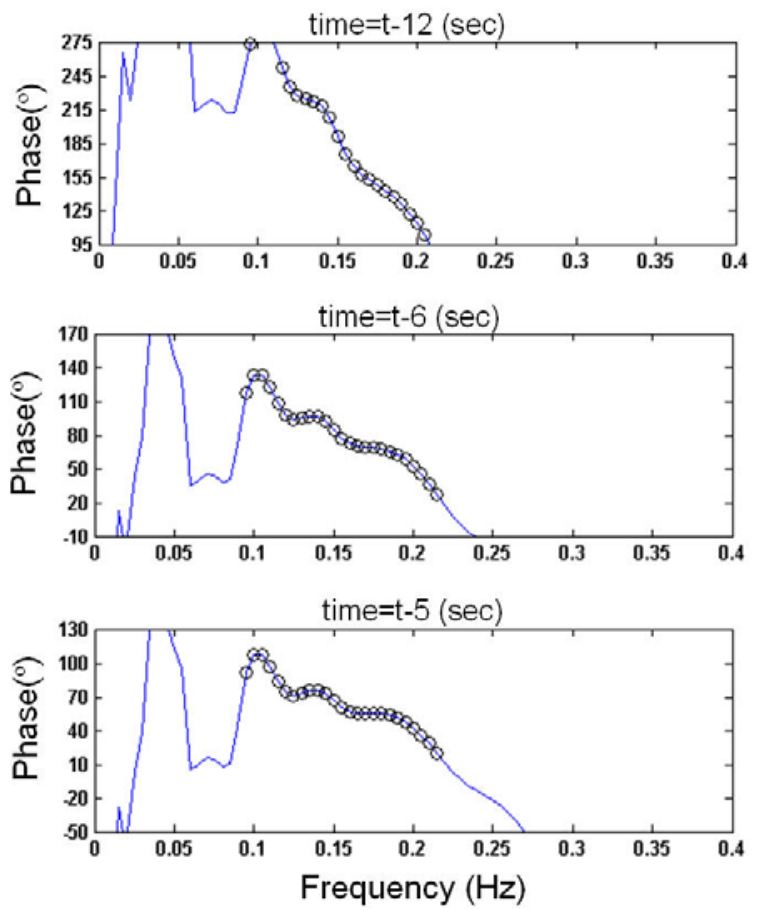

Figure 6. The phase spectrum analyzed by the wavelet algorithm: The instantaneous phase spectrum before the occurrence of freak wave.
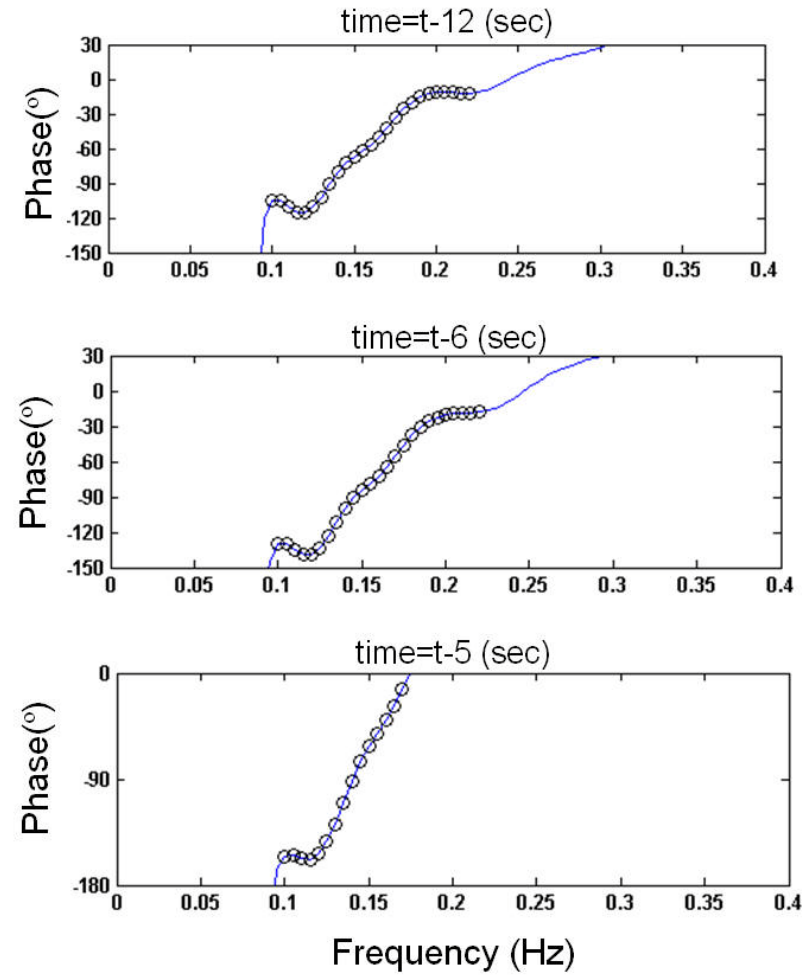

Figure 7. The phase spectrum analyzed by the wavelet algorithm: The instantaneous phase spectrum after the occurrence of freak wave.

\section{SUMMARY}

This study applies the advantage of the fact that the wavelet scalogram simultaneously possesses the energy distributions for time and frequency to investigate the energy characteristics during the 
occurrence of the freak waves. Through analysis of the wavelet phase spectrum and phase spectra, it is found that as freak waves occur, the component waves will lead to constructive superposition due to similar phases. The preliminary results from our study reveal the wavelet transform should be a proper tool to observe instantaneous energy structure from the freak wave events.

\section{ACKNOWLEDGMENTS}

This work was supported by the NSC project (NSC 98-2923-I-006-001-MY4) and Ministry of Education (NCKU Project of Promoting Academic Excellence \& Developing World Class Research Centers). The authors would like to offer their great thanks to these agencies.

\section{REFERENCES}

Buessow, R. 2007. An algorithm for the continuous morlet wavelet transform. Mechanical Systems and Signal Processing, 21, 2970-2979.

Chalikov, D. 2009. Freak waves: Their occurrence and probability, Physics of Fluids, 21, 076602-1 076602-18.

Gramstad, O. and K. Trulsen. 2010. Can swell increase the number of freak waves in a wind-sea? Journal of Fluid Mechanics, 650, 57-79.

Jordan, D., R.W. Miksada, and E.J. Powers. 1997. Implementation of the continuous wavelet transform for digital time series analysis, Review of Scientific Instruments, 68, 1484-1494.

Lee, B.C., L.C. Wu, D.J. Doong, and C.C. Kao. 2007. Seasonal variations of wind and wave data over Taiwan waters, Marine Geophysical Researches, 28, 183-190.

Onorato, M., A.R. Osborne, M. Serio, and S. Bertone. 2001. Freak waves in random oceanic sea states, Physical Review Letters, 86, 5831-5834.

Tucker, M.J. and E.G. Pitt. 2001. Waves in Ocean Engineering, Elsevier Science Ltd, Oxford, 521pp.

Wang, D.W., C.C. Teng, and R. Ladner. 1993. Buoy directional wave measurements using magnetic field components, In: The Second International Symposium on Ocean Wave Measurement and Analysis, 316-329.

Yeh, P.L. and P.L. Liu. 2008. Application of the wavelet transform and the enhanced Fourier spectrum in the impact echo test, NDT \& E International, 41, 382-394. 